\title{
The Evolution of the Solar Magnetic Field
}

\author{
J. Todd Hoeksema \\ W.W. Hansen Experimental Physics Laboratory, Stanford University, 466 Via Ortega, \\ Cypress C13, Stanford, CA 94305, USA \\ email: JTHoeksema@spd.aas.org
}

\begin{abstract}
The almost stately evolution of the global heliospheric magnetic field pattern during most of the solar cycle belies the intense dynamic interplay of photospheric and coronal flux concentrations on scales both large and small. The statistical characteristics of emerging bipoles and active regions lead to development of systematic magnetic patterns. Diffusion and flows impel features to interact constructively and destructively, and on longer time scales they may help drive the creation of new flux. Peculiar properties of the components in each solar cycle determine the specific details and provide additional clues about their sources. The interactions of complex developing features with the existing global magnetic environment drive impulsive events on all scales. Predominantly new-polarity surges originating in active regions at low latitudes can reach the poles in a year or two. Coronal holes and polar caps composed of shortlived, small-scale magnetic elements can persist for months and years. Advanced models coupled with comprehensive measurements of the visible solar surface, as well as the interior, corona, and heliosphere promise to revolutionize our understanding of the hierarchy we call the solar magnetic field.
\end{abstract}

Keywords. Sun: magnetic field, Sun: activity cycles

Of course comprehensively reviewing the evolution of the solar magnetic field in such a short report is impossible, so this discussion focuses on a few of the more salient features of large-scale and long-term evolution. The 22-year magnetic cycle drives most variations of the Sun, from active regions to the corona and heliosphere, and from the polar field reversal to the total solar irradiance. See for example discussions of the most recent deep solar minimum framed by Gibson et al. (2011) and references therein. The Sun's activity is fairly typical for stars of its age and rotation rate, thus what we learn about the drivers of solar dynamo activity will inform and be informed by what we see on other suns.

Figure 1 shows how the axial and equatorial dipole components of the Sun changed during the last 3.5 solar cycles, from 1976-2012, as measured by the Wilcox Solar Observatory. The axial dipole peaks a couple years before the solar minima in 1976, 1986, 1996, and 2009. The equatorial dipole reflects the large-scale average of active regions and peaks more broadly during solar maximum. There is a strong secular trend in the overall amplitude of the dipole, with the most recent extended minimum having less than half the strength of the previous three. The current Cycle 24 and the extended prior minimum is unprecedented in the space age, but is not unlike Cycle 14.

Flux transport models suggest that the photospheric field pattern emerges from the creation and decay of active regions. Active regions drive the most dramatic expressions of the cycle, from flares and coronal mass ejections to particle acceleration and high 


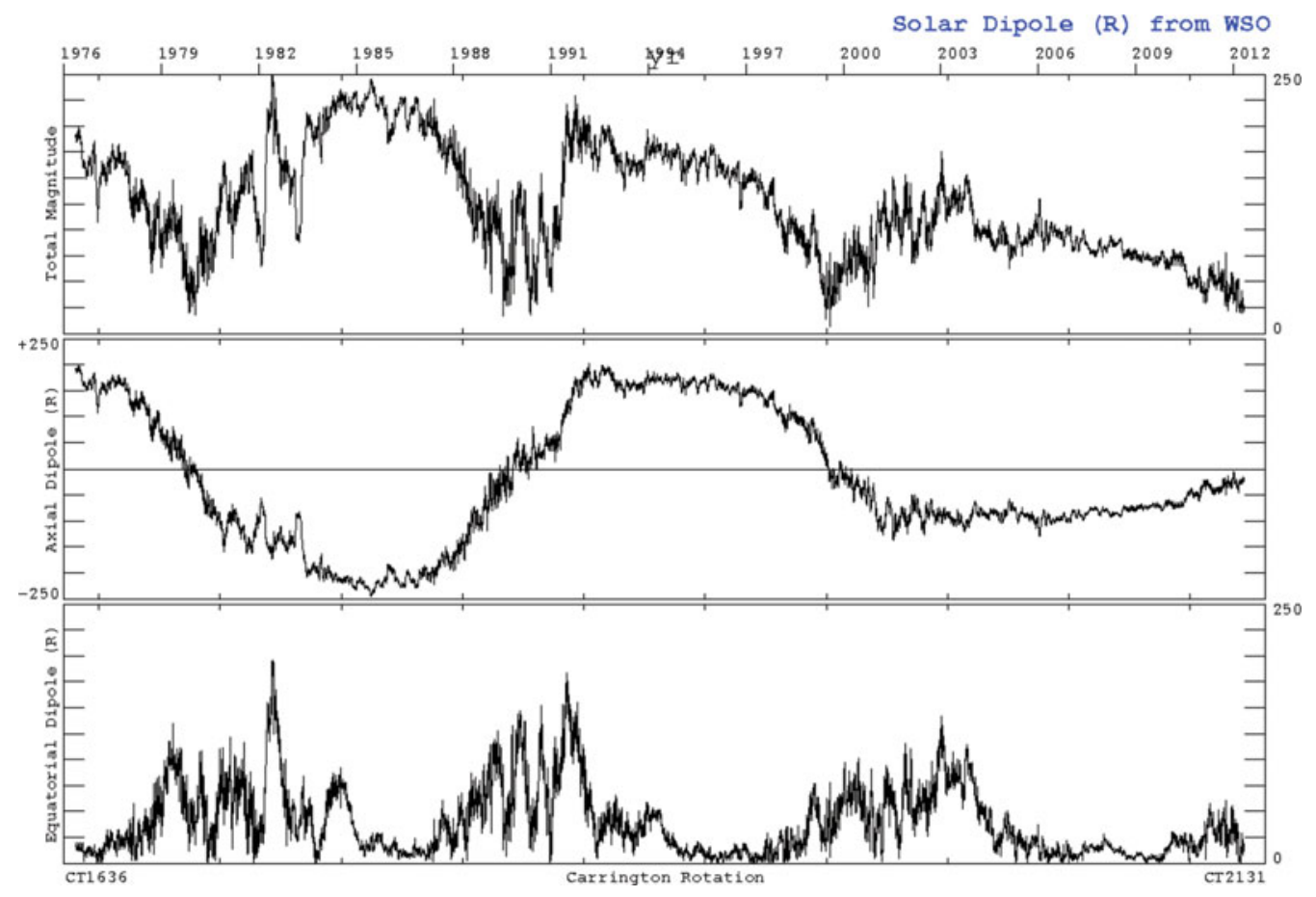

Figure 1. The Sun's dipole field from 1976 - 2012 as observed by the Wilcox Solar Observatory. The top panel shows the total dipole. The center and bottom show the axial and equatorial components. Note the phase of the components relative to the solar cycle and the secular decrease particularly in the axial dipole.

energy radiation. AR 11158 produced the first X-class flare of the cycle at 01:56 UT on February 15, 2011. Figure 2 shows how the region emerged and evolved over 5 days. The complex region demonstrated strong shearing motions and the currents computed from the vector field were strongly peaked at the flare site. The three columns in the figure show the photospheric radial field strength from HMI, the AIA 304A intensity in the overlying atmosphere, and the computed current density. The transverse component of the field increased sharply and permanently at the time of the flare (Sun et al. 2012). The current cycle has so far been weak and this may be related to changes not only in the number and complexity of sunspot groups, but also to an apparent decline in the maximum field strength observed in active regions that cause changes in the longterm relationship between sunspot number and F10.7 cm flux (e.g. Livingston et al. 2012).

As active regions decay, the trailing flux moves poleward, ultimately reversing the dominant magnetic polarity a little after maximum in each hemisphere. Figure 3 shows the decaying magnetic field pattern of a large southern hemisphere spot on July 12, 2012. The red-yellow negative pattern will eventually merge with flux from other regions to reverse the south polar field (e.g. Wang \& Sheeley, 1991; Schrijver \& DeRosa, 2003). The northern hemisphere has been active earlier and will likely have reversed at the end of 2012. Zonally averaging over all longitudes shows how the net flux emerges and moves poleward over the cycle. Figure 4 shows the dominant polarity at high latitude reversing in 2001 as a consequence of surges of flux from decaying low-latitude 


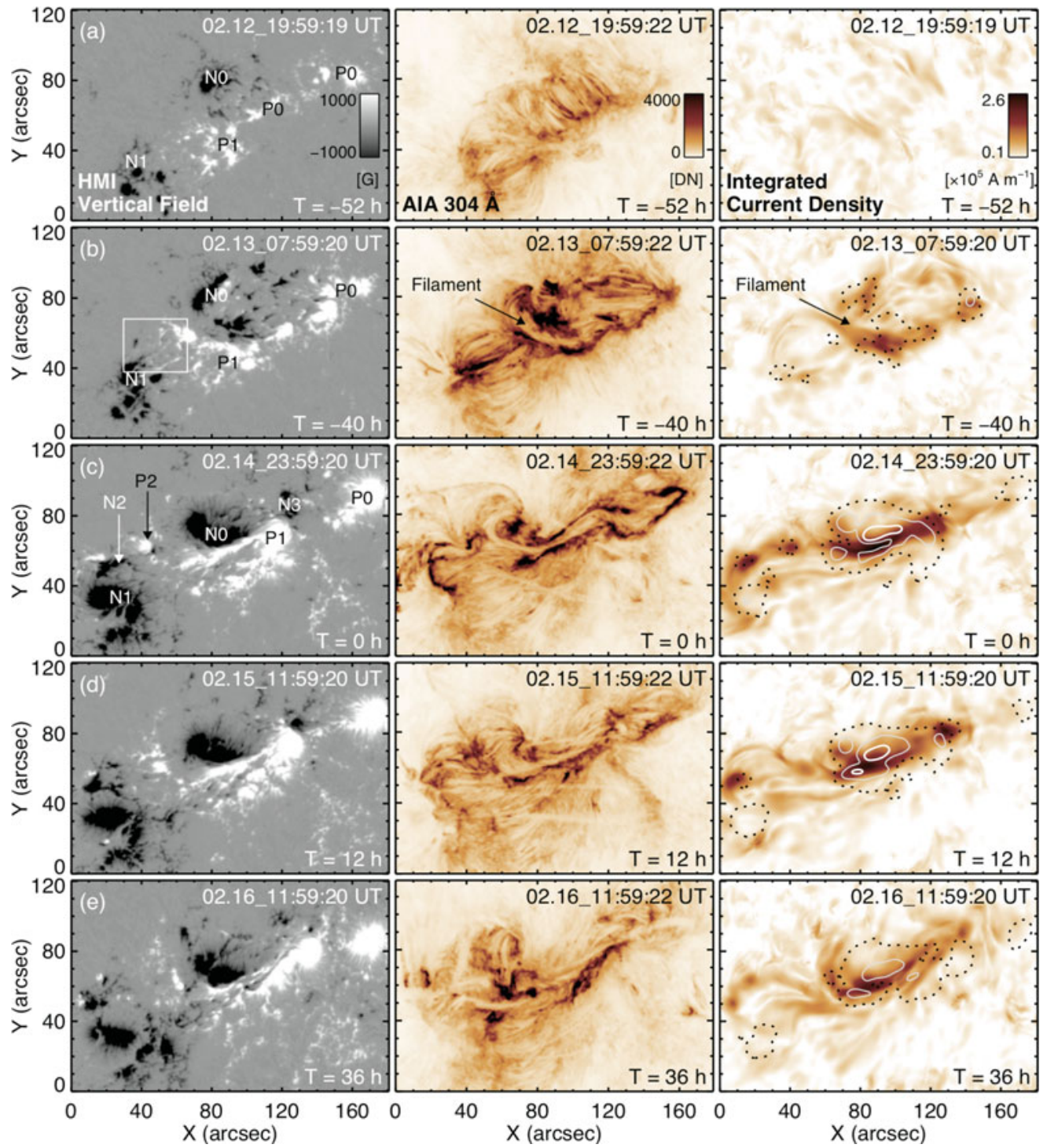

Figure 2. The evolution of the radial component of the magnetic field for AR 11158 from 12-16 February 2011 is shown in the left column. The current density, calculated from the HMI vector field, peaks at about the time of the x-class flare at 01:56 UT on 15 Feb. (right column). The center column shows the simultaneous $304 \mathrm{~A}$ emission in the overlying atmosphere observed by AIA. (Sun et al., 2012).

active regions carried poleward by meridional flows and diffusion. Cycle 24 has begun slowly and the surges are small. Since the polar field was weak, the reversal has proceeded relatively quickly in any case. The peculiarities of the cycle depend on the characteristics of the emerging active regions produced by the solar dynamo and their interactions with the changing flows. How the magnetic dross in the photosphere influences subsequent cycles is unclear. Improving continuous, long-term synoptic observations of the Sun are critical for understand this example of a stellar dynamo. 


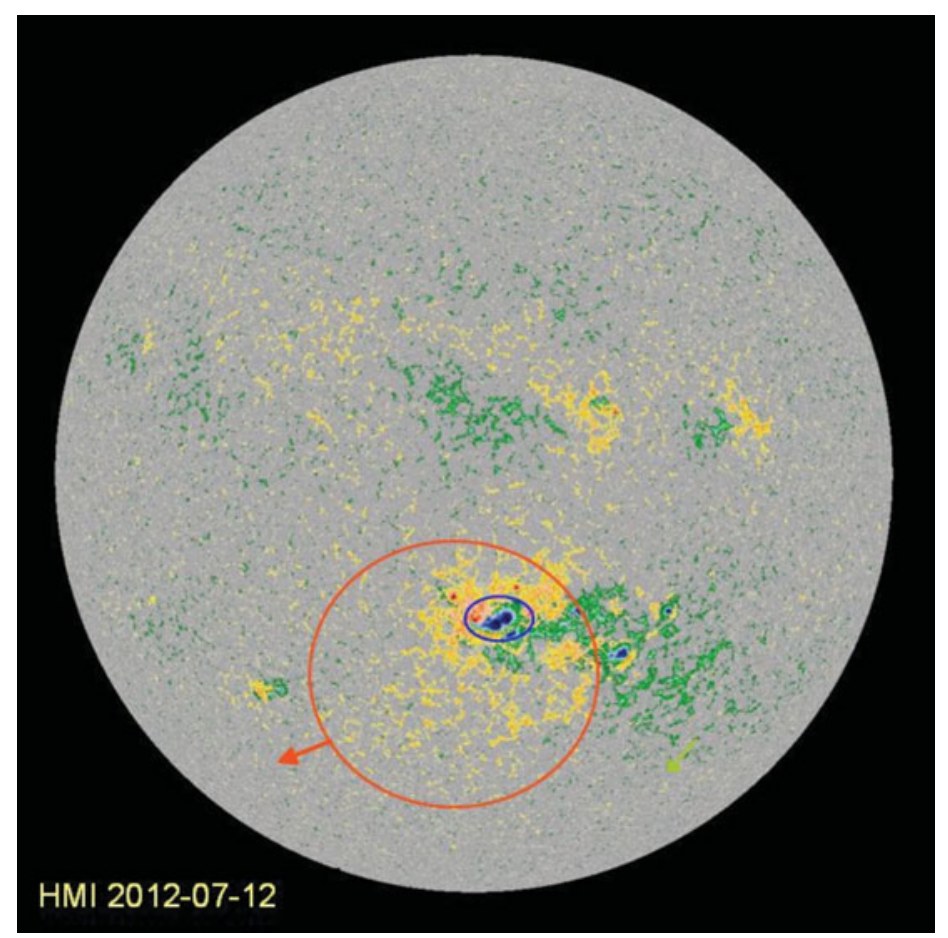

Figure 3. The extensive blue/yellow positive magnetic field region shown in this HMI magnetogram from 12 July 2012 will expand and merge with left-over flux from other decaying active regions and be carried poleward by meridional flows to reverse the southern cap.

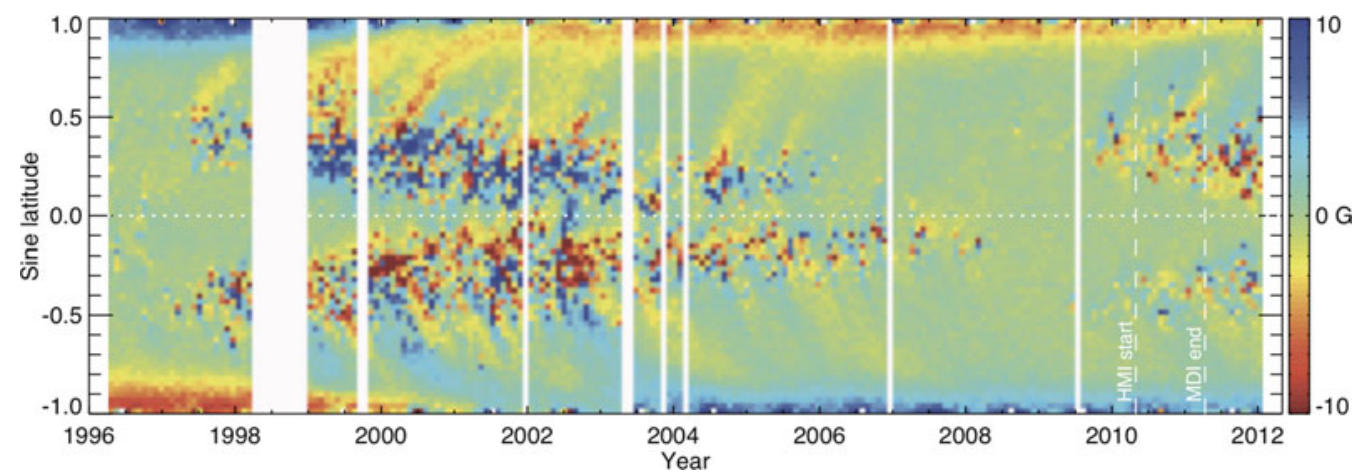

Figure 4. The zonal magnetic field from 1996 - 2012 as observed by MDI and HMI. The net flux in each sine latitude bin is computed for each Carrington Rotation and plotted as a function of time. The positive (blue) north pole reverses in 2001. Surges of following polarity from decaying active regions migrate poleward in 12-18 months to cause the reversal. In the current cycle weaker surges are sufficient to erode the weaker polar field. (Figure courtesy X. Sun)

\section{References}

Gibson, S., et al. 2011, Solar Phys. 274, 5

Livingston, W., Penn, M., \& Svalgaard, L. 2012, ApJL 757, 8

Schrijver, C. J. \& DeRosa, M. 2003, Solar Phys. 212, 165

Sun, X., et al. 2012, ApJ 748, 77

Wang, Y.-M., Sheeley, N., et al. 1991, ApJ 375, 761 